\title{
Eigenenergies of 3D-Coulomb and 3D-Harmonic Oscillator Potentials from WKB Quantization: Point Canonical Transformation
}

\author{
Hosung Sun \\ Department of Chemistry, Sunghythkwan Lnwersity, Sun on 4+0-746, Korea. E-mail: hsuna skkuedu \\ Received September 28, 2007
}

\begin{abstract}
A direct application of the WKB quantization to the three-dimensional Coulomb potential does not yield the exact eigenenergies. The three-dimensional Coulomb potential is converted to a Morse potential by using the point canonical transformation. Then the WKB quantization is applied to the Morse potential to find a relationship between the eigenenergies of the Coulomb and those of the Morse potentials. From the relationship the exact eigenenergis of the Coulomb potential are determined. The same method is found to be also valid for the three-dimensional harmonic oscillator potential. And the Langer modified WKB quantization is algebraically derived.
\end{abstract}

Key Words : WKB quantization, Coulomb potential, Hamonic oscillator potential

\section{Background}

The Schrödinger equation for one-dimensional syrstem is (in units of $2 m=\hbar=1$ )

$$
\left[-\frac{d^{2}}{d r^{2}}+V(r)\right] \Psi_{n}(r)=E_{n} \Psi_{n}(r)
$$

Here we consider a potential $V(r)$ that varies smoothly i.e. there exist two classical turning points for all bound states. The lowest order Wentzel-Kramers-Brillouin approximation (or the WKB quantization condition) for the above sy'stem is 1.2

$$
\int_{r_{L}}^{r_{R}} \sqrt{E_{n}-V(r)} d r=\left(n+\frac{1}{2}\right) \pi n=0.1 .2 \ldots
$$

where $r_{L}$ and $r_{R}$ are inuer (short distance) and outer (long distance) turning point, respectively. i.e. $V\left(r_{L}\right)=V\left(r_{R}\right)=E_{\text {st }}$. The WKB quantization is a semiclassical method of obtaining approximate eigenenergies in the limiting case of large quantum numbers or high energy. But it is found to be exact for the one-dimensional harmonic oscillator potential and the one-dimensional Morse potential.$^{2-4}$ For other potentials. for example. the 3D-Coulomb potential or the 3D-harmonic oscillator potential the WKB quantization does not reproduce the exact eigenenergies.

Sukhatme et al. reported that all solvable potentials are inter-related to each other by a certain transformation. 56 Ther classify the solvable potentials by type-I and type-II. The type-II category includes three potentials. i.e. the onedimensional Morse $\left(V^{\mathrm{M}}(r)\right)$, the 3D-Coulomb $\left(V^{(r}(r)\right)$ and the 3D-harmonic oscillator $\left(V^{\mathrm{H}}(r)\right)$ potentials. And the three potentials can be transformed to each other by the so-called point canonical transformation.

In this work we briefly introduce a new method of determining the exact eigenenergies of $V^{C}(r)$ from the point canonical transformation and the WKB quantization. As mentioned before. the exact eigenenergies of $V^{\mathrm{M}}(r)$ can be obtained from the WKB quantization but the eigenenergies of $V^{C}(r)$ are not. First. we transform the Sclurödinger equation with $V^{C}(r)$ into another Schrödinger equation with $V^{\mathrm{M}}(r)$. And then we apply the WKB quantization to the $V^{\mathrm{M}}(r)$ to obtain a relationship between the eigenenergies of $V^{C}(r)$ and those of $V^{\mathrm{M}}(r)$. Since the exact eigenenergies of $V^{\mathrm{M}}(r)$ are known. the eigenenergies of $V^{C}(r)$ can be determined from the relationship. The above argument is also valid for the $3 \mathrm{D}$-harmonic oscillator potential $V^{\mathrm{H}}(r)$.

We define the 3D-Coulomb and the 3D-harmonic oscillator potentials as follows. ${ }^{4-6}$ For a given angular momentum quantum number $l$, the $3 \mathrm{D}$-Coulomb potential $(q=$ charge parameter) is

$$
V(r)=V^{C}(r)=-\frac{q^{2}}{r}+\frac{l(l+1)}{r^{2}} \quad(0<r<\infty)
$$

and the eigenenergies $E_{n}^{C}=-\frac{q^{4}}{4(n+1+1)^{2}}$ for $n=0,1,2, \ldots$

Since $V^{C}(r)$ goes to zero as $r \rightarrow \infty$. the bound state eigenenergies $E_{n}^{C}$ are negative.

For a given $/$, the 3D-harmonic oscillator potential $(\omega=$ frequency parameter) is

$$
V(r)=V^{\mathrm{H}}(r)=\frac{1}{4} \omega^{2} r^{2}+\frac{l(l+1)}{r^{2}} \quad(0<r<\infty)
$$

and the eigenenergies $E_{n}^{\mathrm{H}}=\left(2 n+l+\frac{3}{2}\right) \omega$ for $n=0,1,2, \ldots$

For reference the one-dimensional Morse potential $(A, B$. $\alpha=$ parameters) is

$$
I^{\mathrm{M}}(x)=B^{2} e^{-2 \alpha x}-2 B\left(A+\frac{\alpha}{2}\right) e^{-\alpha x} \quad(-\infty<x<\infty) .
$$

The phase-space integral in the WKB quantization can be analytically evaluated as ${ }^{4}$ 


$$
\int_{x_{L}}^{x_{R}} \sqrt{E_{n}^{\mathrm{M}}-V^{\mathrm{M}}(x)} d x=\frac{\pi}{\alpha}\left(A+\frac{\alpha}{2}-\sqrt{-E_{n}^{\mathrm{M}}}\right)
$$

where $V^{M 1}\left(x_{L}\right)=V^{M 1}\left(x_{R}\right)=E_{n}^{M}$. Since $V^{M 1}(x)$ goes to zero as $x \rightarrow \infty$, the eigenenergies $E_{n}^{\mathrm{M}}$ are negative.

\section{Canonical Transformation and WKB for 3D-Coulomb Potential}

For the 3D-Coulomb potential the Schrödinger equation is

$$
\left[-\frac{d^{2}}{d r^{2}}-\frac{q^{2}}{r}+\frac{l(l+1)}{r^{2}}\right] \Psi_{n}^{C}(r)=E_{n}^{C} \Psi_{n}^{C}(r) \quad(0<r<\infty)
$$

with the boundary condition of $\Psi_{n}^{\mathrm{C}}(\mu)=0$ as $r \rightarrow 0$ and $r \rightarrow \infty$. Canonically transforming, i.e.. changing the variable $r$ to $x=-\ln r$ and the wave function $\Psi_{, f}(r)$ to $\Phi_{n}(x)=e^{x / 2} \Psi_{n}^{c}(r)$, one obtains

$$
\begin{gathered}
{\left[-\frac{d^{2}}{d x^{2}}-E_{n}^{c} e^{-2 x}-q^{2} e^{-x}+\left(I+\frac{1}{2}\right)^{2}\right] \Phi_{n}(x)=0} \\
(-\infty<x<\infty) .
\end{gathered}
$$

The new function $\Phi_{n}(x)$ satisfies the boundary condition of $\Phi_{n}(x)=0$ as $x \rightarrow-\infty$ and $x \rightarrow \infty$. The transformation is sometimes called the Langer transformation. ${ }^{7.9}$ Note that the canonical transformation converts $\mathrm{Eq}$. (9) on the half-line (0. $\infty)$ to $\mathrm{Eq} .(10)$ on the full-line $(-\infty . \infty)$.

Once $E_{s}$ and $\Phi_{n}(x)$ are given. Eq. (10) can be considered as a Schrödinger equation for a Morse potential

$$
\begin{gathered}
V^{\mathrm{M}}(x)=-E_{n}^{C} e^{-2 x}-q^{2} e^{-x} \text { with } E_{n}^{\mathrm{M}}=-\left(1+\frac{1}{2}\right)^{2} \text {. i.e. . } \\
{\left[-\frac{d^{2}}{d x^{2}}+V^{M}(x)\right] \Phi_{n}(x)=E_{n}^{M 1} \Phi_{n}(x) .}
\end{gathered}
$$

Eq. (11) looks awkward because the potential contains the ndependent $E_{n}^{C}$ term. But, in fact. the potential is not a function of $n$ because the potential is defined for a fixed $n$. i.e. Eq. (11) is defined for each $n$. Note that the eigenvalue $E_{n}^{\mathrm{M}}$ is the same for all $n$. Therefore Eq. (11), as a whole. is a Sclurödinger equation with the eigenfuncion $\Phi_{n}(x)$ and the eigenenergy $E_{n}^{\mathrm{M}}$. $^{10}$

One can evaluate $E_{n}^{\mathrm{M}}$ in Eq. (11) using the WKB quantization in Eq. (2) that is exact for Morse potentials.

$$
\int_{x_{I}}^{x_{R}} \sqrt{E_{n}^{\mathrm{M}}-\left(-E_{n}^{(?} e^{-2 x}-q^{2} e^{-x}\right)} d x=\left(n+\frac{1}{2}\right) \pi
$$

where $V^{\lambda 1}\left(x_{L}\right)=V^{M 1}\left(x_{R}\right)=E_{n}^{M}$. (In Appendix we explicitly show why the WKB quantization is exact for Morse potentials but not exact for 3D-Coulonb potentials or for 3D-harmonic oscillator potentials.) Comparing the potential in Eq. (12) with that in Eq. (7), one immediately finds that $\alpha=1, B=\sqrt{-E_{n}^{c}}$, and $A=q^{2} / 2 \sqrt{-E_{n}^{c}}-1 / 2$. From the phase-space integral expression in Eq. (8) and the WKB quantization in $\mathrm{Eq}$. (12). one obtains

$$
\pi\left(\frac{q^{2}}{2 \sqrt{-E_{n}^{C}}}-\sqrt{-E_{n}^{\mathrm{M}}}\right)=\left(n+\frac{\mathrm{l}}{2}\right) \pi \text { for } n=0,1,2, \ldots
$$

And the Morse eigenenergy $E_{n}^{\mathrm{M}}$ is

$$
E_{n}^{\mathrm{i}}=-\left(\frac{q^{2}}{2 \sqrt{-E_{n}^{\mathrm{C}}}}-n-\frac{1}{2}\right)^{2} .
$$

Eq. (14) shows the relationship between the eigenenergies of the original 3D-Coulomb potential $-q^{2} / r+l(l+1) / r^{2}$ and those of the transformed Morse potential $-E_{n}^{C} e^{-\hat{\lambda} x}-q^{2} e^{-x}$. Since $E_{n}^{\mathrm{M}}=-(l+1 / 2)^{2}$. Eq. (14) gives the eigenenergies of the 3D-Coulomb potential $E_{n}^{C}=-q^{+} / 4(n+1+1)^{2}(n=0$. $1.2, \ldots$ ) that is identical with that in Eq. (4). Indeed the new method that utilizes the point canonical transformation and the WKB quantization reproduces the exact eigenenergies of the 3D-Coulomb potential.

To make the WKB quantization exact for the 3D-coulomb potential. modified versions of WKB quantization have been suggested. The earliest work was the Langer modification where the centrifugal term $l(l+1) / r^{2}$ in Eq. (19) is replaced by $(l+1 / 2)^{2} / r^{2}$. Though various modifications or improvements of the WKB quantization. since the Langer's work. have been studied they all include the term $(l+1 / 2)^{2} / r^{2}$ (or some operator related to it) in their modification. ${ }^{8.9 .11}$

The Langer modification has its own physical origin. but using our transformation teclunique we can justify the existence of the modification algebraically. Inserting $E_{n}^{\mathrm{M}}=-(1+1 / 2)^{2}$ into the WKB quantization in Eq. (12). one obtains

$$
\int_{x_{L}}^{x_{R}} \sqrt{-\left(l+\frac{1}{2}\right)^{2}+E_{n}^{C} e^{-2 x}+q^{2} e^{-x}} d x=\left(n+\frac{1}{2}\right) \pi .
$$

Changing the variable $x$ back to $r$ using the previous $x=$ -lnt; one obtains

$$
\int_{r_{L}}^{r_{R}} \sqrt{E_{n}^{\mathrm{C}}-\left\{-\frac{q^{2}}{r}+\frac{(l+1 / 2)^{2}}{r^{2}}\right\}} d r=\left(n+\frac{1}{2}\right) \pi .
$$

Eq. (16) is none other than the Langer modified WKB quantization that can exactly reproduce the eigenenergies of the 3D-Coulomb potential. We would like to stress that our transformation teclunique naturally introduces the Langer modification without imposing any semiclassical approximation.

\section{Example of 3D-Harmonic Oscillator Potential}

The method proposed in the previous section can be applied to any potential that can be transformed to a Morse potential. In this section. taking the 3D-hamonic oscillator potential as another example. we repeatedly show how the method works.

For the 3D-harmonic oscillator potential. the Schrödinger equation is 


$$
\begin{aligned}
{\left[-\frac{d^{2}}{d r^{2}}+\frac{1}{4}\left(l^{2} r^{2}+\frac{l(l+1)}{r^{2}}\right]\right.} & \Psi_{n}^{\mathrm{H}}(r)=E_{n}^{\mathrm{H}} \Psi_{n}^{\mathrm{H}}(r) \\
& (-\infty<x<\infty) .
\end{aligned}
$$

Changing the variable $r$ to $x=-2 \ln r$ and the wave function $\Psi_{n}^{\mathrm{H}}(r)$ to $\Phi_{n}(x)=e^{x / 4} \Psi_{n}^{\mathrm{H}}(r)$, one obtains

$$
\left[-\frac{d^{2}}{d x^{2}}+\frac{(1)^{2}}{16} e^{-2 x}-\frac{E_{n}^{\mathrm{H}}}{4} e^{-x}+\frac{1}{4}\left(1+\frac{1}{2}\right)^{2}\right] \Phi_{n}(x)=0 .
$$

Eq. (18) can be considered as a Schrödinger equation for a Morse potential $V^{\lambda \mathrm{I}}(x)=\left(\omega^{2} / 16\right) e^{-2 x}-\left(E_{n}^{\mathrm{H}} / 4\right) e^{-x}$ with eigenenergy $E_{n}^{M 1}=-(1 / 4)(l+1 / 2)^{2}$.

The WKB quantization for Eq. (18) is

$$
\int_{x_{L}}^{x_{R}} \sqrt{E_{n}^{\mathrm{M}}-\left(\frac{\omega^{2}}{16} e^{-2 x}-\frac{E_{n}^{\mathrm{H}}}{4} e^{-x}\right)} d x=\left(n+\frac{1}{2}\right) \pi .
$$

Comparing the Morse potential in Eq. (19) with that in $\mathrm{Eq}$. (7), one finds that $\alpha=1 . B=(0) / 4$. and $A=E_{n}^{\mathrm{M}, 1} / 2(1)-1 / 2$. From Eq. (8) and Eq. (19). one obtains

$$
\pi\left(\frac{E_{n}^{\mathrm{H}}}{2 \omega}-\sqrt{-E_{n}^{\mathrm{M}}}\right)=\left(n+\frac{1}{2}\right) \pi \text { for } n=0,1,2 \ldots
$$

Then the Morse eigenenergy $E_{n}^{\mathrm{M}}$ is

$$
E_{n}^{\mathrm{M}}=-\left(\frac{E_{n}^{\mathrm{H}}}{2 \omega}-n-\frac{1}{2}\right)^{2} .
$$

Since $E_{n}^{\mathrm{M}}=-(1 / 4)(l+1 / 2)^{2}$. the eigenenergies of the 3D-harmonic oscillator potential is $E_{n}^{\mathrm{H}}=(2 n+1+3 / 2)(n)$ $(n=0,1,2 \ldots)$ that is identical with the exact eigenenergies in Eq. (6). Again we are able to derive the exact eigenenergies of the 3D-harmonic oscillator potential using the point canonical transformation and the WKB quantization.

Following the same way for the 3D-Coulomb potential. the Langer modified WKB quantization is derived as

$$
\int_{r_{L}}^{r_{R}} \sqrt{E_{n}^{\mathrm{H}}-\left\{\frac{1}{4} \omega^{2} r^{2}+\frac{(l+1 / 2)^{2}}{r^{2}}\right\}} d r=\left(n+\frac{1}{2}\right) \pi .
$$

The Langer modification for the 3D-harmonic oscillator potential turns out to be the same as that for the 3DCoulomb potential. Actually it is the same for all solvable spherically symmetric potentials. ${ }^{9.12}$

\section{Conclusion}

The point canonical transformation has been introduced to explain the inter-relation among the exactly solvable potentials. ${ }^{5.6}$ This transformation has been utilized to explain the supersymumetry ${ }^{6,13}$ of certain potentials. ${ }^{11}$ In this work we have found another interesting usage of the transformation.

Using the point canonical transformation. the Schrödinger equation for the 3D-Coulomb potential is converted to another Schrödinger equation with a Morse potential. Then an application of the WKB quantization to the Morse potential yields a relationship between the eigenenergies of the Coulomb and those of the Morse potentials. From the relationship the exact eigenenergies of the Coulomb potential are derived. The Langer modified WKB quantization is also algebraically derived. The proposed method is valid for any potential that can be transfonmed to a Morse potential. As another example, the exact eigenenergies of the 3Dharmonic oscillator potential are determined by using the same method.

Other solvable potentials belonging to the type-I category (e.g. the Eckart potential. the Scarf potential, and the RosenMorse potential, etc.) are related to the type-II potentials (e.g. the Morse potential, etc.) through redefinition of parameters and the so-called limiting procedure. ${ }^{5}$ Therefore the type-I potentials are easily transformed to a Morse potential and, consequently. our method can be again used to determine the exact eigenenergies of the type-I potentials. As a matter of fact, all the type-I and type-II potentials have a common feature of Natanzon potentials that are exactly solvable.

One cannot say that the WKB quantization is exact for all solvable potentials. However it is not appropriate for one to assert that the WKB quantization is useless when one desires exact eigenenergies of solvable potentials other than the Morse potential (or one-dimensional harmonic oscillator potential.) As shown in this work. the WKB quantization may be useful even in low energy regime where semiclassical approximation is not valid

\section{Appendix}

Another way of modifying the WKB quantization is to introduce the nonintegral Masloy index $u^{2.14}$ In this scheme the exact quantization condition is written as

$$
\int_{r_{2}}^{r_{n}} \sqrt{E_{n}-r^{(r)}} d r=\left(n-\frac{\mu \lambda}{4}\right) \pi
$$

When the Maslov index $\mu=2$. Eq. (Al) is, of course, reduced to the WKB quantization. There is no general way of evaluating the Maslov index for an arbitrary potential. However it can be evaluated for exactly solvable potentials. The direct method of evaluating the Masloy index is to adopt an exact quantization. for esample. the analytical transfer matrix method (ATMM).

The ATMM quantization condition can be summarized as ${ }^{15}$

$$
\int_{i}^{r} \sqrt{E_{n}-I^{\prime}(r)} d r-\delta_{n}=(n+1) \pi .
$$

And the so-called scattering-led phase shift $\hat{\phi}_{i}$ is

$$
\delta_{n}=-\int_{r_{c}}^{r_{c}} k_{n}{ }^{\prime}(r) \frac{P_{n}(r)}{P_{n}{ }^{\prime}(r)} d r
$$

where $k_{n}{ }^{\prime}(r)=\frac{d\left[\sqrt{E_{n}-I^{\prime}(r)}\right]}{d r}$ and $P_{n}^{\prime}(r)=\frac{d P_{n}(r)}{d r}$

$P_{n}(r)$ is the minus log derivative of the $n$-th state wave function $\Psi_{n}(r)$. i.e.. $P_{\mathrm{n}}(r)=\left(d \Psi_{\mathrm{n}}(r): d r\right) \Psi_{\mathrm{n}}(r)$. Comparing Eq. (Al) with Eq. (A2). one immediately finds that the Maslow index $a t=4\left(1-\hat{\delta}_{n}: \pi\right)$

For the general form of Morse potential given in Eq. (7) including the forms in Eqs. (12) and (19), we have found $\delta_{n}=1 / 2 \pi$ in our fommer work. ${ }^{16}$ Consequently the Maslow index,$a$ is 2 . It proves that 
the WKB quantization should be valid for the Morse potential.

In our previous paper ${ }^{1:}$ in this Journal we have presented $\delta_{n}=(\sqrt{l(t+1)}-t) \pi$ for the 3D-Coulomb potential in Eq. (3). Therefore the Maslov index $t$ is $4[l-1-\sqrt{l(l+1)}]$. Using the same method suggested in the previous paper we algebraically evaluate the phase shift and the Maslov inder for the 3D-hamonic oscillator potential Eq. (5) for the first time. And the result is that $\delta_{n}=[(\sqrt{I(l-1)}-I) 2-14] \pi$ and $\mu=3-2 \sqrt{l(I+1)}-2 l$. (In Ref. 14. based on qualitative arguments. the same Maslov index has been deduced.) It shows that the WKB quantization is not walid for the 3D-Coulomb potential nor for the 3D-harmonic oscillator potential since " $/$ is not 2 . However, this work extends the validity of the WKB quantization to the 3D-Coulomb and 3D-harmonic oscillator potentials through the point canonical transformation.

\section{References}

1. Fröman, N.: Fröman, P. O. JHKB Approximation; North Holland: Amsterdam. 1965.
2. Friedrich, H.: Trost. J. Plys. Rep. 2004. $397,359$.

3. Ivanow. I. A. J. Phys A. Hoh Gen. 1997.30.3977.

4. Hruška. M.: Keung. W.-Y.: Sulhatme. U. Phtus. Rev A 1997. 55. 3345 .

5. Gangopadhyaya, A.; Panigrahi, P. K.; Sukhatme, U. P. Helv Phus. Acta 1994. 67. 363.

6. Cooper. F.: Khare. A.: Sukhatme. U. P. Plns. Rep. 1995. 251. 267.

7. Langer. R. E. Phws. Rev 1937.51.669

8. Krieger. J. B.: Rosenzweig. C. Plys. Rev 1967, 16t, 171

9. Sergeento, M. N. Phys. Rev. A 1996, 53. 3798.

10. Haymaker. R. W. Rau, A. R. P. Am. J. Plys. 1986. 54.928

11. Bruev. A. S. Pho Lett. A 1992. 161.407.

12. Ou. Y.: Cao. Z.: Shen. Q. Phus Lett .1 2003. 318. 36.

13. Sun1. H. Bull. Korean Chem. Soc. 2006. 27. 515.

14. Friedrich, H.; Trost. J. Plys. Ren A 1996. 5t, 1136.

15. Ou. Y. C. Cao. Z: Shen, O. J. Chen. Phns. 2004. 121.8175.

16. Sun. H. Plys. Lett \& 2005. 338. 309 .

17. Sun1. H. Bill. Korean Chem. Soc. 2007. 28. 408. 